\title{
Functionalization of PU Foams via Inorganic and Organic Coatings to Improve Cell and Tissue Interactions
}

\author{
Serena Bertoldi 1,2, Nicola Contessi Negrini ${ }^{2}$, Andrea Cochis ${ }^{3,4}$, Francesca Uberti 5 , \\ Maria Cristina Tanzi ${ }^{2}$ and Silvia Farè ${ }^{1,2, *}$ \\ 1 Department of Chemistry, Materials and Chemical Engineering “G. Natta”, Politecnico di Milano, \\ Via Mancinelli 7, 20131 Milano, Italy; serena.bertoldi@polimi.it \\ 2 INSTM, National Consortium of Materials Science and Technology, Local Unit Politecnico di Milano, \\ Piazza Leonardo da Vinci 32, 20131 Milano, Italy; nicola.contessi@polimi.it (N.C.N.); \\ mariacristina.tanzi@polimi.it (M.C.T.) \\ 3 Department of Health Sciences, Università del Piemonte Orientale, Via Solaroli 17, 28100 Novara, Italy; \\ andrea.cochis@med.uniupo.it \\ 4 Center for Translational Research on Autoimmune \& Allergic Diseases-CAAD, Corso Trieste 15/A, \\ 28100 Novara, Italy \\ 5 Department of Translational Medicine, Università del Piemonte Orientale, Via Solaroli 17, 28100 Novara, \\ Italy; francesca.uberti@med.uniupo.it \\ * Correspondence: silvia.fare@polimi.it
}

Received: 14 February 2019; Accepted: 15 February 2019; Published: 26 February 2019

\begin{abstract}
In this work an innovative method to obtain hybrid bio-functional scaffolds has been developed. Polyether urethane (PU) foam scaffolds were synthetized by one-step gas foaming process. PU foams were coated with crosslinked gelatin hydrogel to promote cell adhesion and proliferation for the regeneration of soft tissues (e.g., adipose tissue). PU foams were coated with inorganic coating (i.e., $\mathrm{CaPs}$ ) to improve the interaction with osteoblasts for bone tissue regeneration. The functionalized 3D PU porous scaffolds have been characterized investigating morphological properties by SEM and microCT, water uptake and coating stability, and compressive mechanical properties. Adipose tissue derived stem cells (ADSCs), endothelial cells (MS1), amnion mesenchymal cells (AMCs) and chorion mesenchymal cells (CMCs) isolated from human placenta were in vitro cultured on the hybrid functionalized 3D scaffolds. Mechanical properties showed elastic modulus ranging between $15.75 \pm 2.14$ and $22.9 \pm 3.1 \mathrm{kPa}$; in vitro biological studies showed good cell adhesion, proliferation, and differentiation. In particular, compared to the results with uncoated PU, when cells where differentiated into adipocytes, Oil red O staining confirmed a higher presence of lipid droplets; in case of osteoblasts differentiation, inorganic extracellular matrix deposition was evidenced on CaPs coated PU. The obtained results suggest the important role of an adequate coating on the scaffold to stimulate a better interaction with cells, promoting the differentiation into different cells phenotypes.
\end{abstract}

Keywords: polyurethane foam; 3D scaffold; coating; crosslinked gelatin; CaPs; micro-CT; mechanical characterization; in vitro cytocompatibility

\section{Introduction}

Regeneration of human tissues by a tissue engineering approach requires the design and fabrication of engineered scaffolds able to support the regrowth of pathological/missing tissues. The fabricated scaffolds should possess specific properties to guarantee a correct tissue regeneration, 
including adequate porosity, biomimetic mechanical properties, biocompatibility and optimal surface properties to promote cell colonization and tissue infiltration after the in vivo implantation [1]. A possible strategy to achieve the required properties is to fabricate hybrid scaffolds that are composed by a structural biomaterial, acting as scaffold, coated by a second bioactive material that improves the surface properties of the scaffold. To do this, either inorganic or organic coatings can be used depending on the target tissues to be regenerated and on the desired surface properties to be achieved.

For instance, inorganic coatings can be performed with calcium phosphates or hydroxyapatite, mainly used to target bone regeneration. In fact, inorganic coatings of a scaffold mimic the bone inorganic component; moreover, the inorganic coating can guide stem cell fate towards osteogenic phenotype and promote the scaffold's osteoconductivity. Among others, electrospun hydroxyethyl cellulose nanofibers coated with calcium phosphate showed improved tensile properties and cells adhesion [2]; 3D printed polycaprolactone scaffolds coated with calcium phosphate showed enhanced osteogenic properties [3]; electrospun poly(L-lactic acid) coated by electrophoretic deposition of calcium phosphates showed to be able to mimic the bone components and microstructure [4].

Alternatively, organic coatings, such as gelatin hydrogel coatings, can be used to promote the cell-biomaterial interactions of scaffolds that lack cell-adhesive motifs, thus promoting cells adhesion and tissue colonization. For instance, PLGA porous scaffolds coated with gelatin demonstrated improved hydrophilicity, mechanical properties and in vitro proliferation of mesenchymal stem cells [5]. Similarly, electrospun nanofibrous PCL scaffolds coated with gelatin showed ECM biomimetic properties and improved cells adhesion [6].

In this work, we describe the use of polyurethane foams as scaffolding materials since they exhibit promising properties for tissue engineering purposes but, as many other synthetic polymers, they lack surface bioactive properties [7,8]. Thus, we designed the coating of polyurethane foams either by using calcium phosphates, to target the regeneration of bone tissue, or gelatin hydrogels, to target the regeneration of soft tissues. We optimized a versatile vacuumassisted coating system to obtain hybrid polyurethane scaffolds and we subsequently investigated morphological, mechanical and in vitro biological properties of the fabricated hybrid scaffolds.

\section{Materials and Methods}

\subsection{Materials}

All reagents and solvents were purchased from Sigma Aldrich (Milan, Italy), unless otherwise specified. 4-4'-Methylene diphenyl diisocyanate prepolymer and polyols used for the polyurethane synthesis were gently provided by Bayer, Leverkusen, Germany. Distilled water was purchased from Idrochimica Srl (Fizzonasco, Italy).

\subsection{Polyurethane Foam Synthesis}

The PU foam was synthesized by gas foaming following an optimized procedure described elsewhere $[9,10]$. PU foams were synthesized by one-step bulk reaction; the following reagents were used: MDI prepolymer (Desmodur ${ }^{\circledR} \mathrm{PF}$, Bayer), an ad hoc poly-ether-polyol mixture, FeAcetylacetonate (FeAA) as catalyst and water $\left(2 \% w / w_{\text {polyol }}\right)$ as expanding agent. The reagents were mixed together and put to react in a closed, custom-made polymethylmethacrylate (PMMA) mould (Figure 1a) in order to achieve the highest homogeneity of pores dimension and distribution. Afterwards, the foam was cut into sheets $(h=3 \mathrm{~mm})$ with a metal saw, and manually punched to obtain cylindrical samples $(\varnothing=10 \mathrm{~mm})$.

\subsection{Gelatin Hydrogel Synthesis}

To coat all surfaces throughout the PU foam, a gelatin hydrogel (GEL) was prepared by following an optimized crosslinking reaction [11,12], based on a Michael-type addition. Briefly, a water solution of type A gelatin from porcine skin $(6 \%$ and $15 \% w / v)$ was crosslinked with 
methylenebisacrylamide (MBA), using an equimolar amount with respect to primary amino groups present in gelatin.

\subsection{Hybrid Scaffold Preparation}

Hybrid PU_GEL and PU_CaP scaffolds were prepared using a home-made vacuum chamber (Figure $1 b$ ) to promote the coating of the PU foam by penetration of the gelatin hydrogel or CaPs particles suspension inside the PU porous structure. The system is composed by a cylindrical PMMA chamber $\left(h=9 \mathrm{~cm}, \varnothing_{\mathrm{int}}=6.5 \mathrm{~cm}\right)$ sealed on the top with a PMMA lid with a silicon O-ring. Inside the chamber, a PMMA ring hanger $\left(\varnothing_{\mathrm{ext}}=6 \mathrm{~cm}\right)$ is held at a height of $5.5 \mathrm{~cm}$. The ring hanger can hold up to 6 steel hangers to fix the PU foam samples during the coating with gelatin or CaPs. The chamber is connected to a vacuum pump by a Pyrex tube equipped with a valve.

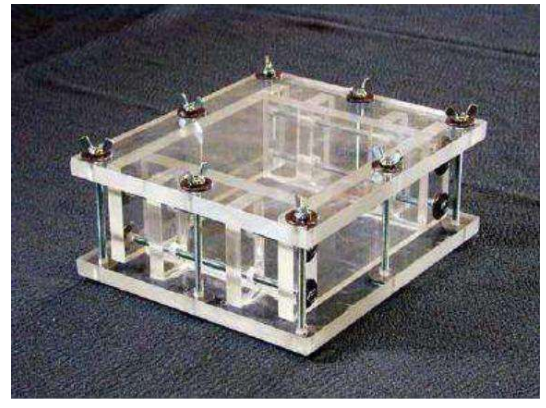

(a)

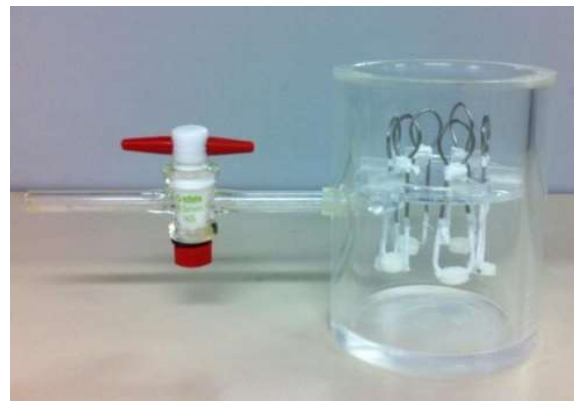

(b)

Figure 1. (a) PMMA mold used for the synthesis of the PU foams; (b) Custom-made PMMA cylindrical chamber.

PU_GEL samples were prepared in the vacuum chamber by immersing PU samples $(n=6)$ in a gelatin aqueous solution $(6 \%$ or $15 \% w / v)$ with MBA crosslinker, applying repeated vacuum cycles $(n=6)$ to allow for the substitution of the air in PU foam pores with the gelatin solution. At the end of the sixth cycle, the coated PU samples (PU_GEL) were removed from the gelatin solution and put at $50{ }^{\circ} \mathrm{C}$ for $24 \mathrm{~h}$ to allow the complete gelatin hydrogel crosslinking. After that, PU_GEL samples were purified in pure ethanol for $24 \mathrm{~h}$, distilled water for $24 \mathrm{~h}$ and dehydrated by immersion in $50 \%$ ethanol for $2 \mathrm{~h}$ and pure ethanol for $2 \mathrm{~h}$. Dehydrated samples were finally stored under vacuum before further use. PU_GEL6 and PU_GEL15 were obtained, using a gelatin solution with a gelatin concentration respectively of $6 \%$ and $15 \% w / v$.

PU_CaP samples were prepared following the procedure described for the PU_GEL samples. Briefly, a CaPs suspension ( $4 \% w / v \beta$-TCP in distilled water) was put in the PMMA chamber with a magnet to homogenize the suspension under stirring $(800 \mathrm{rpm})$. After closing the chamber with the sailing cap, the valve was opened and the vacuum pump was turned on; vacuum was maintained for $2 \mathrm{~h}$, then the CaP coated samples were removed from the chamber and left under hood to dry.

\subsection{Morphological Characterization}

PU, PU_GEL, and PU_CaP samples were observed with a stereomicroscope (WILD M8, Heerbrugg, Switzerland), and a scanning electron microscope (SEM, Cambridge Instrument Stereoscan 360, London, UK) to qualitatively estimate the presence of gelatin or CaPs particles on the pore walls, and assess the homogeneity of the coating distribution, both onto surface and cross section of the samples. Before SEM observation, the specimens were sputter-coated (Edwards Sputter Coater 5150B, Bergshill, UK) with a thin gold layer. The samples were also analyzed using an 1172 microCT imaging system (Skyscan ${ }^{\circledR}$, Aartselaar, Belgium) desktop X-ray CT scanner at $5.99 \mu \mathrm{m}$ voxel resolution, X-ray tube current $226 \mu \mathrm{A}$, voltage $44 \mathrm{kV}$ and power of $10 \mathrm{~V}$ without any filters. Open porosity, pore interconnection, and average pore size were evaluated by microCT. 


\subsection{Water Uptake}

Water uptake test at $37^{\circ} \mathrm{C}$ was performed on the specimens $(n=3)$ of the different materials. At first, the dry weight $\left(w_{0}\right)$ was measured, then specimens were put in a 24 multiwell plate, and each well was filled with $2 \mathrm{~mL}$ of distilled water. Samples were weighted $\left(w_{i}\right)$ at several time points $(t=15,30 \mathrm{~min}, 1,2,3,6,24 \mathrm{~h}$ and every $24 \mathrm{~h}$ until plateau or coating degradation was detected). The water uptake $(\% \mathrm{WU})$ was calculated using the following formula:

$$
\mathrm{WU}[\%]=\frac{w_{i}-w_{0}}{w_{0}} \times 100
$$

\subsection{Mechanical Characterization}

Compression mechanical tests were performed in strain rate control mode using a Dynamic Mechanical Analyzer (DMA Q800, TA Instruments, New Castle, DE, USA). The experiments were carried out on 5 specimens for each considered samples. Samples were hydrated in distilled water for $72 \mathrm{~h}$, until the \%WU plateau was reached. After an isotherm at $37^{\circ} \mathrm{C}$ for $5 \mathrm{~min}$, a loading run was performed with a strain ramp at $-2.5 \% / \mathrm{min}$, until reaching of $50 \%$ deformation, followed by an unloading run (strain ramp $=5 \% / \mathrm{min}$, until $0.1 \%$ deformation).

\subsection{In vitro Cytocompatibility Tests}

In vitro cytocompatibility tests were assessed using different cells depending on the target application of the coated scaffold. For scaffold coated with crosslinked gelatin (PU_GEL6, PU_GEL15) murine adipose tissue derived stem cells (ADSCs), isolated from visceral adipose tissue and endothelial cells (MS1, ECACC 09111802) were selected. In particular, ADSCs were differentiated after seeding into adipocytes, while MS1 were considered to investigate the possible stimulation of angiogenesis driven by the gelatin hydrogels. Then, the evaluation of proliferation and possible differentiation to osteoblastic phenotype of amnion mesenchymal cells (AMCs) and chorion mesenchymal cells (CMCs), isolated from human placenta, was investigated on PU_CaP foams.

For ADSCs, -MEM supplemented with penicillin/streptomycin (P/S, 1\% v/v) and L-glutamine $\left(\begin{array}{ll}1 \% & v / v\end{array}\right)$ was used; adipogenic differentiation was induced $24 \mathrm{~h}$ after seeding using DMEM, additioned with fetal bovine serum (FBS, $10 \% v / v)$, dexamethasone $\left(0.392 \times 10^{-3} \mathrm{~g} / \mathrm{L}\right)$, insulin $\left(10^{-3} \mathrm{~g} / \mathrm{L}\right)$, 3-Isobutyl-1-methylxanthine (IBMX, $\left.55.56 \mathrm{~g} / \mathrm{L}\right)$, and indomethacin (14.31 g/L). MS1 cells were cultured in DMEM-HAM's F12 culture medium, supplemented with FBS (10\% v/v), Lglutamine $(1 \% v / v)$, and P/S solution $(1 \% v / v)$. AMCs and CMCs were cultured in EMEM and Nonhematopoietic OsteoDiff Medium (NH OsteoDiff Medium).

Cells were cultured using the cells density reported in Table 1, together with the considered time-points and the analysis performed at each timepoint.

Table 1. Cells density, time-points and analysis performed on the scaffolds seeded with the different cells phenotypes.

\begin{tabular}{cccc}
\hline Scaffolds & Cells & Cell Density & Analysis \\
\hline \multirow{2}{*}{ PU, PU_GEL6, PU_GEL15 } & ADSCs & $1 \times 10^{3}$ cells/scaffold & SEM, XTT, Oil Red O staining \\
& MS1 & $1 \times 10^{3}$ cells/scaffold & XTT \\
\hline \multirow{2}{*}{ PU, PU_CaP } & AMCs & $5 \times 10^{6}$ cell $\mathrm{mL}^{-1}$ & SEM, hematoxylin/eosin staining \\
& CMCs & $5 \times 10^{6}$ cell mL $\mathrm{mL}^{-1}$ & SEM \\
\hline
\end{tabular}

\subsection{Statistical Analysis}

Each experiment was repeated at least on three replicates and all results were expressed as mean and standard deviation. Statistical analysis was performed using one-way ANOVA test (GraphPad Prism 6.0 Software). The statistical difference between two groups of data was considered to be significant for $p<0.05$. 


\section{Results and Discussion}

\subsection{Morphological Characterization}

\subsubsection{SEM Observation}

\section{PU_GEL6 and PU_GEL15}

A uniform gelatin coating was observed by SEM onto the pore walls of PU_GEL6 (Figure 2a) and PU_GEL15 samples (Figure 2b); the cross-sections evidenced that the 3D porous structure was completely interpenetrated with the gelatin hydrogel (Figure 2f,g). Uncoated PU showed a uniform pore distribution with a good pore interconnection (Figure $2 \mathrm{c}, \mathrm{h}$ ).

\section{PU_CaP}

Compared to the uncoated PU foam (Figure 2e,1), CaPs were detected both on the surface and cross-section (Figure 2d,i), evidencing good interconnections among the pores, apparently without no other important differences.

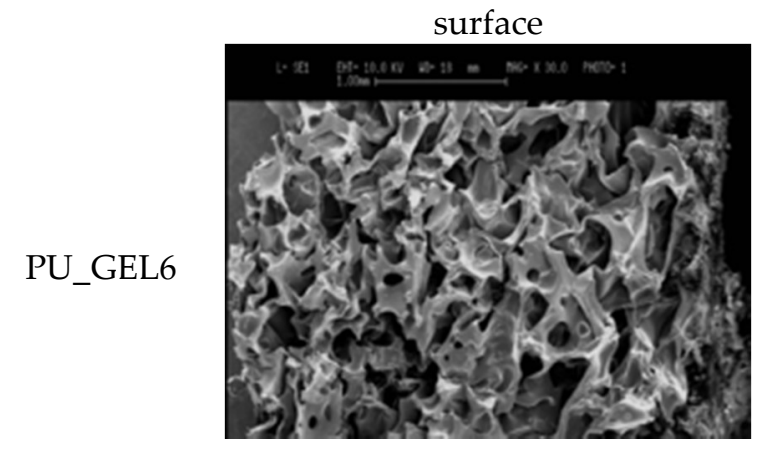

(a)

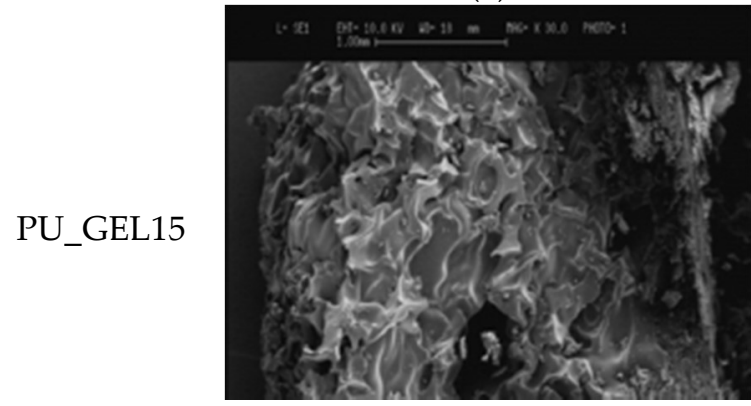

(b)

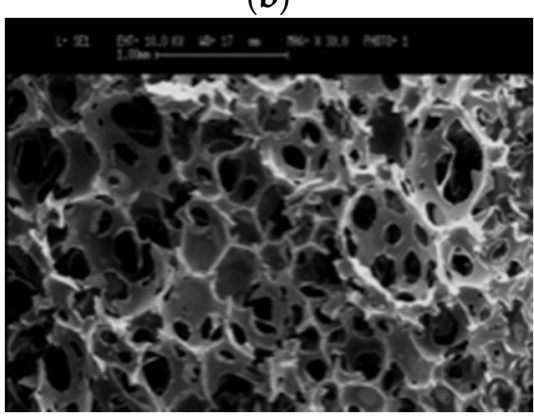

(c)

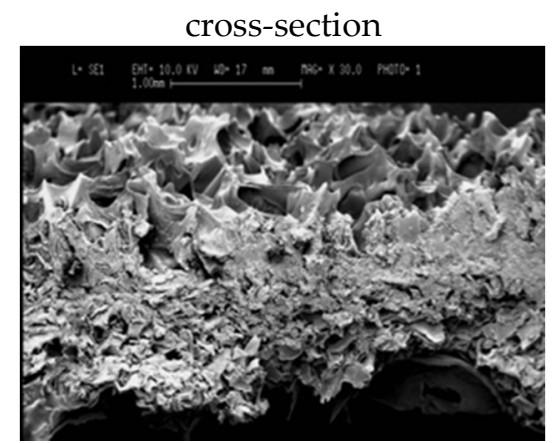

(f)

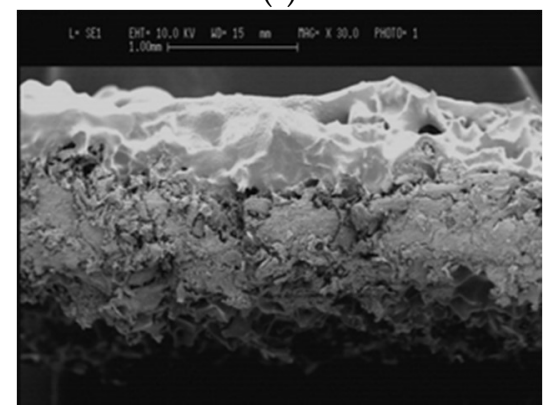

(g)

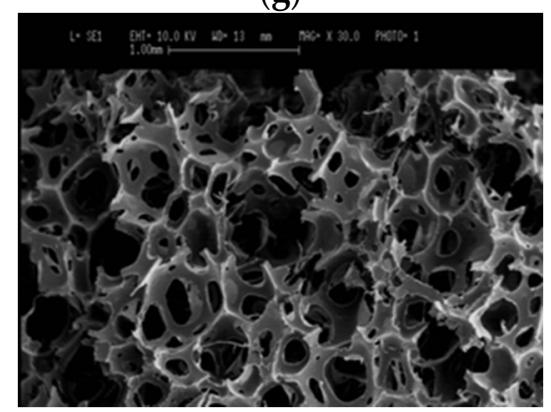

(h) 


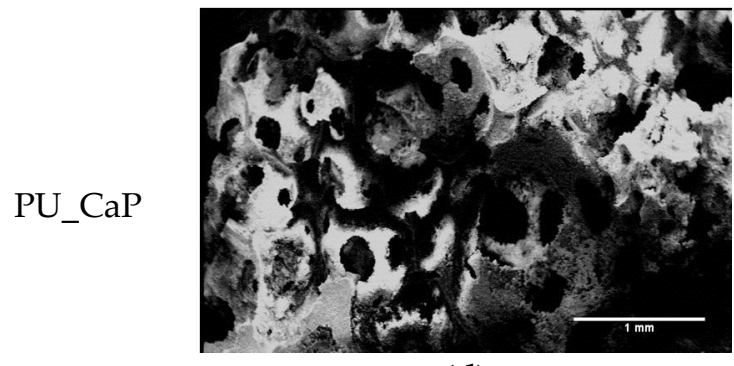

(d)

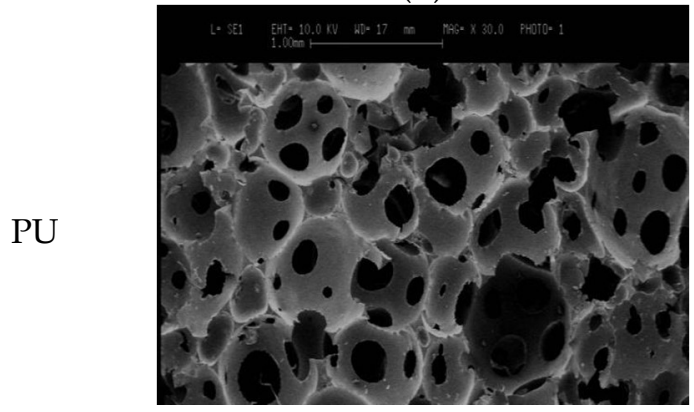

(e)

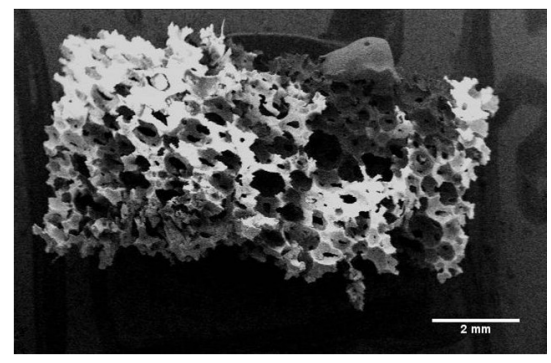

(i)

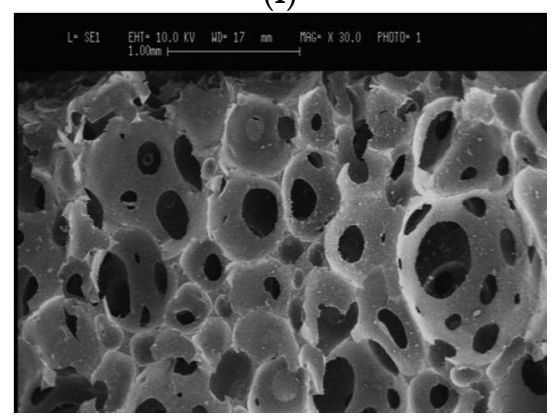

(1)

Figure 2. SEM images of the coated and uncoated PU foam. Samples coated with crosslinked gelatin hydrogel: (a) surface of PU_GEL6; (b) surface of PU_GEL15; (f) cross-section of PU_GEL6; and (g) cross-section of PU_GEL15. Control uncoated PU samples: (c) surface and (h) cross-section. Samples coated with CaPs: (d) surface of PU_CaP; (i) cross-section of PU_CaP. Control uncoated PU: (e) surface and (i) cross-section. Scale bar: $1 \mathrm{~mm}$, scale bar (h): $2 \mathrm{~mm}$.

\subsubsection{Micro-CT}

\section{PU_GEL6 and PU_GEL15}

Micro-CT was performed on coated and uncoated PU foams to better evidencing the influence of gelatin hydrogels on pore interconnections. In particular, the analysis was performed on samples in dry (Figure 3a-c) and hydrated condition (Figure 3d-f); the percentage of open porosity is reported in Table 3. The differences among the samples are statistically different $(p<0.05)$; the percentage of open porosity decreases importantly increasing the gelatin concentration, in dry and hydrated condition.

dry

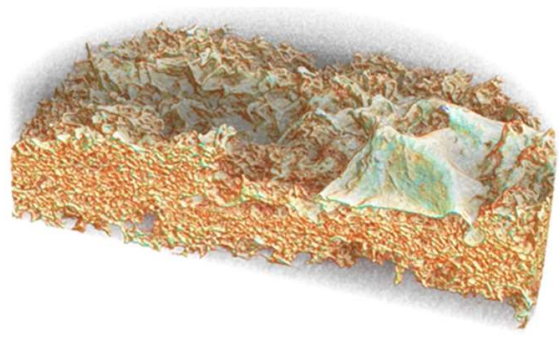

(a)

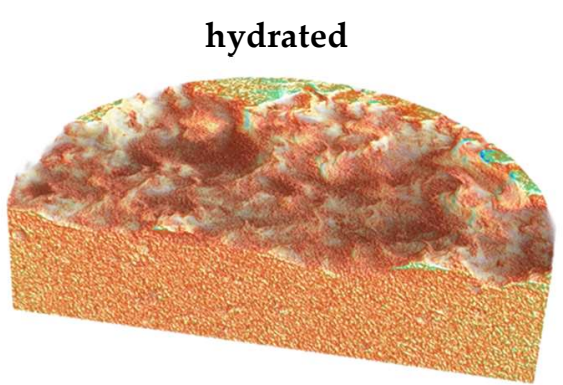

(d) 
PU_GEL15

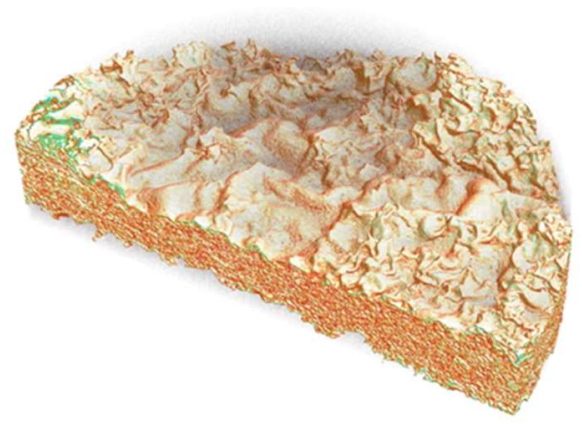

(b)

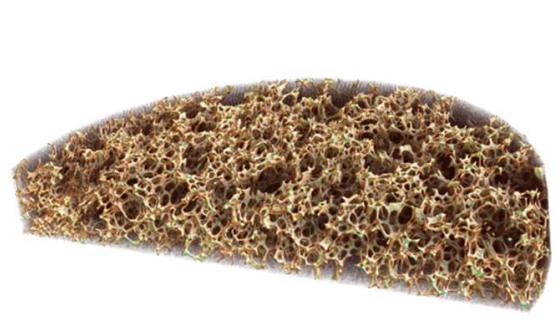

(c)

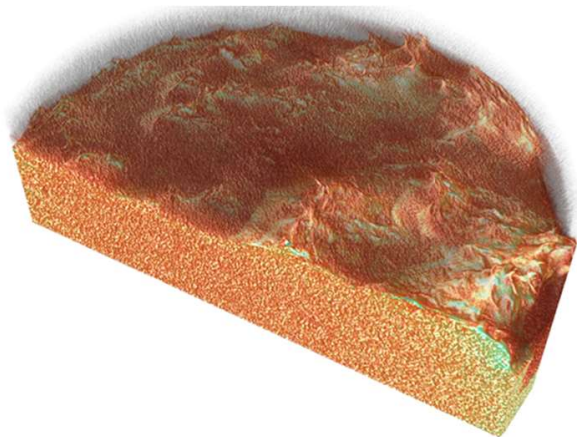

(e)

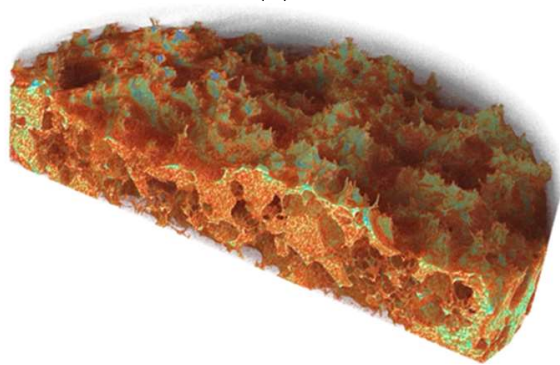

(f)

Figure 3. Micro-CT 3D models of the PU foam. PU coated with crosslinked gelatin hydrogel, in dry condition: (a) PU_GEL6; (b) PU_GEL15; and (c) uncoated PU. PU coated with crosslinked gelatin hydrogel in hydrated condition: (d) PU_GEL6; (e) PU_GEL15; and (g) uncoated PU.

Table 3. Open porosity values (\%) of PU foam samples uncoated and coated with crosslinked gelatin hydrogel or CaPs. For PU_GEL and control PU the open porosity was evaluated in dry and hydrated condition.

\begin{tabular}{ccccc}
\hline Condition & PU_GEL6 & PU_GEL15 & PU_CaP & PU \\
\hline dry & $49.73 \pm 0.49$ & $24.25 \pm 6.69$ & $85.19 \pm 0.90^{(\wedge)}$ & $91.26 \pm 1.78$ \\
hydrated & $13.14 \pm 1.26$ & $5.15 \pm 2.45$ & - & $45.23 \pm 1.68$ \\
\hline
\end{tabular}

(^): For PU_CaP scaffold the value of open porosity was evaluated by a gas picnometer in dry condition.

\subsubsection{Water Uptake}

\section{PU_GEL6 and PU_GEL15}

The water uptake and degradation kinetics for PU uncoated and coated with crosslinked gelatin hydrogel were evaluated up to 30 days. The WU plateau was reached after $6 \mathrm{~h}$ and stabilized after $24 \mathrm{~h}$ for all samples; statistical differences $(p<0.05)$ were evidenced when comparing control PU with PU_GEL6 and PU_GEL15, and PU_GEL_6 with PU_GEL15. Control PU and PU_GEL6 swelled more (WU 451\% and 328\%, respectively) than PU_GEL15 (203\% WU). All samples remained stable (i.e., no degradation of the gelatin coating) up to 30 days of incubation in distilled water (Figure 4a).

\section{PU_CaP}

CaPs-coated foam exhibited a lower water uptake compared to control PU foam (184\% vs. $266 \%$, respectively, Figure 4b). The WU\% for PU_CaP increased rapidly until reaching the plateau after only $15 \mathrm{~min}$, showing a water uptake kinetic significantly different (up to $24 \mathrm{~h}$ ) from that of control PU. Therefore, the CaPs coating enhanced the initial WU of PU scaffold due to the higher hydrophilicity provided by the CaPs. 


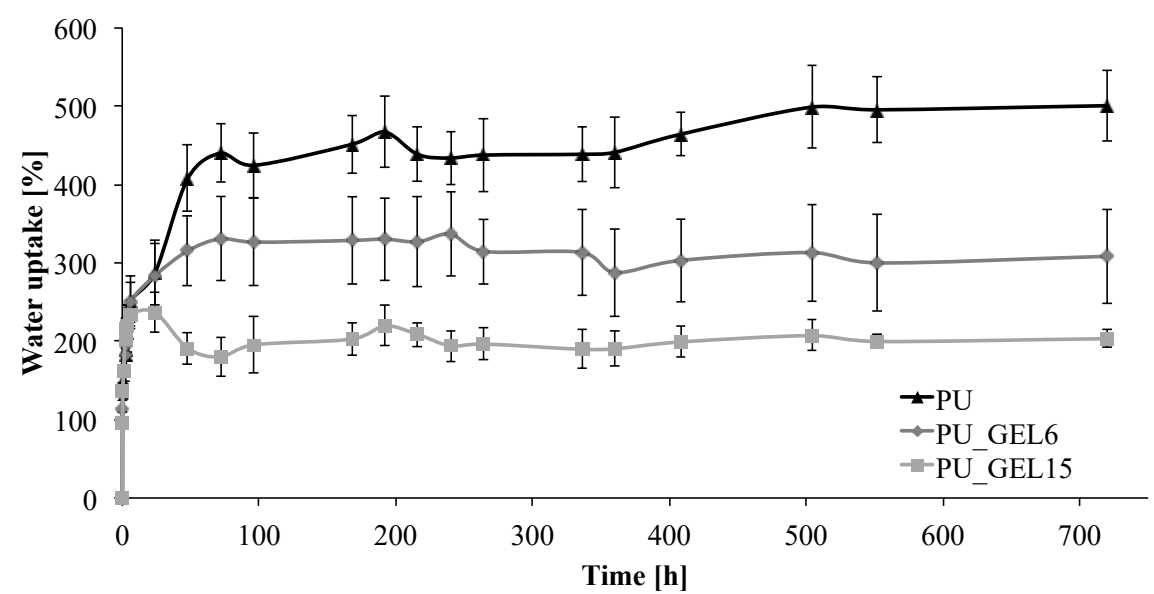

(a)

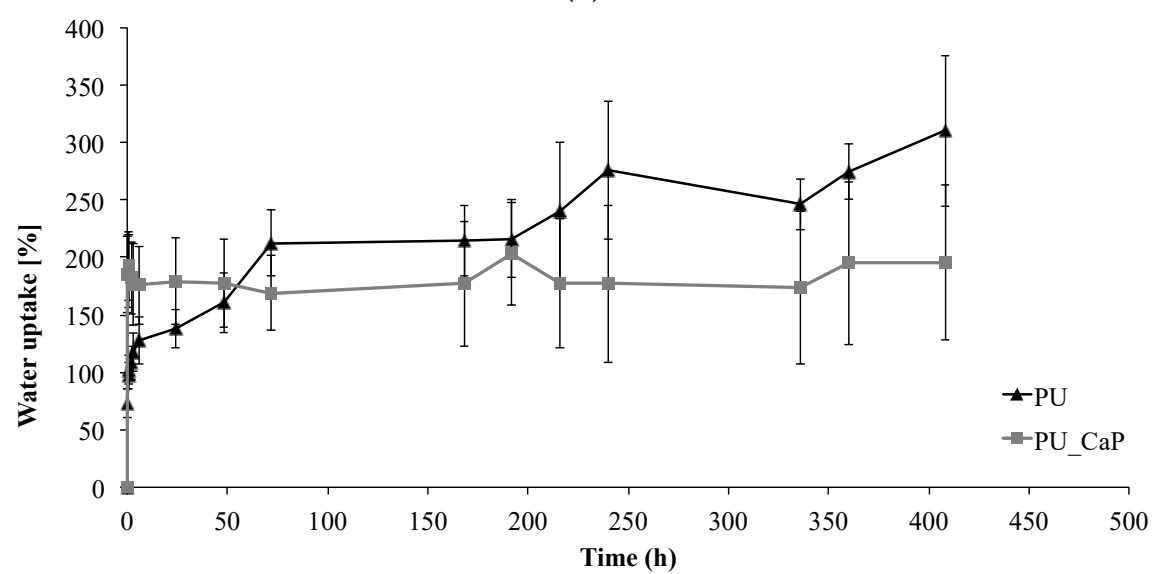

(b)

Figure 4. Water uptake (WU\%) kinetics for (a) PU coated with crosslinked gelatin hydrogels and (b) PU coated with CaPs. Uncoated PU was considered as control scaffold.

\subsection{Mechanical Characterization}

Mechanical tests were performed on coated PU under compression run down to $-50 \%$ strain followed by an unloading run up to $0 \%$ strain. Uncoated PU was also tested as control.

\section{PU_GEL6 and PU_GEL15}

Figure 5a and Table 4 report representative stress/strain curves and the values of the considered mechanical parameters. It is possible to observe that the stress/strain behavior of gelatin coated-PU was comparable to that of control PU. This is caused by the fact that the mechanical properties of the scaffold are mainly influenced by the PU foam, and the gelatin hydrogel, at both concentrations, did not influence the compression behavior. In particular, PU_GEL6 exhibited a behavior more similar to that of control PU than PU_GEL15. The mechanical parameters highlighted no significant difference between the three scaffolds, confirming the low contribution of the gelatin coating to the mechanical properties of the PU_GEL scaffolds.

\section{PU_CaP}

PU_CaP scaffold did not show an improvement in the mechanical properties of the PU foam, as demonstrated by the similarity of the representative stress/strain curves reported in Figure $5 b$. The values of the mechanical parameters (Table 4) did not exhibit any significant difference comparing PU_CaP and uncoated PU. The presence of CaPs on the surface of the pore walls is aimed to improve the interaction of the scaffold with cells that can recognize the CaP coating as similar to the natural inorganic phase in bone. 


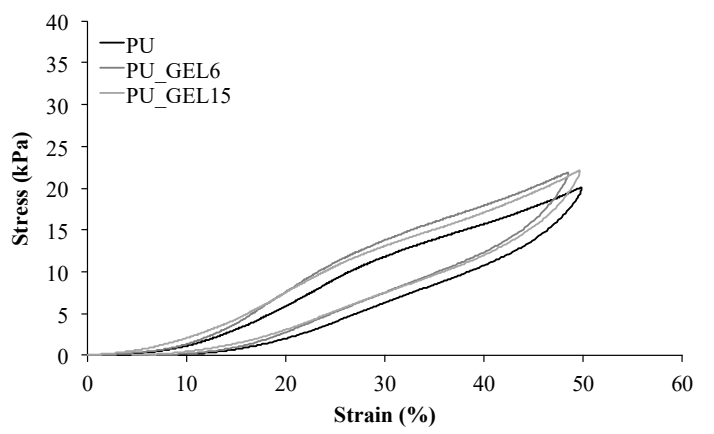

(a)

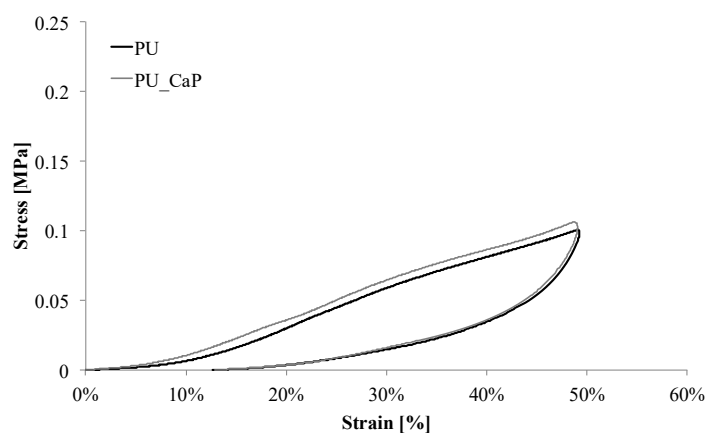

(b)

Figure 5. Representative stress/strain curves for the compression tests performed on the PU_GEL (a) and PU_CaP (b) scaffolds. Uncoated PU was considered as control.

Table 4. Values of the considered mechanical parameters for PU_GEL and PU_CaP scaffolds. Uncoated PU was considered as control.

\begin{tabular}{cccccc}
\hline Samples & $\boldsymbol{E} \mathbf{( k P a )}$ & $\boldsymbol{R}_{\mathbf{1 0 - 3 0}} \mathbf{( k P a )}$ & $\boldsymbol{\delta}_{\max }(\mathbf{k P a})$ & $\varepsilon_{\text {res }}(\mathbf{\%})$ & $\boldsymbol{I}\left(\mathbf{J} / \mathbf{d m}^{3}\right)$ \\
\hline PU_GEL6 & $7.51 \pm 2.46$ & $56.60 \pm 2.28$ & $22.60 \pm 1.82$ & $6.16 \pm 0.49$ & $1.8 \pm 0.12$ \\
PU_GEL15 & $8.13 \pm 2.19$ & $53.17 \pm 6.89$ & $22.98 \pm 3.08$ & $5.64 \pm 1.11$ & $1.8 \pm 0.15$ \\
PU & $7.09 \pm 2.34$ & $51.81 \pm 8.41$ & $19.44 \pm 2.99$ & $6.56 \pm 0.65$ & $1.6 \pm 0.31$ \\
PU_CaP & $82 \pm 4$ & $295 \pm 40$ & $120 \pm 20$ & $10.9 \pm 1.5$ & $16.2 \pm 2$ \\
PU & $63 \pm 22$ & $282 \pm 56$ & $100 \pm 20$ & $11.9 \pm 1.3$ & $14 \pm 3$ \\
\hline
\end{tabular}

\subsection{In vitro Cytocompatibility Tests}

\section{PU_GEL6 and PU_GEL15}

PU coated with gelatin hydrogel and control PU were seeded with ADSCs and MS1 to investigate the possibility to promote adipocyte differentiation and angiogenesis.

Figure 6a reports ADSCs viability values onto the considered scaffolds. It is possible to observe that cell viability was higher than $60 \%$ at each time-point. The scaffold did not show any significant differences increasing the time $(p>0.05)$. At Day 1, PU_GEL15 showed lower cell viability $(p<0.05)$ compared to PU, but after 3 days of culture, the viability values were comparable. Oil Red O staining was performed on PU coated with gelatin hydrogel (Figure 6c), qualitatively observing differentiation of ADSCs in adipocytes.

Figure $6 \mathrm{~b}$ shows XTT results for MS1 cells cultured on the considered scaffolds. The viability of endothelial cells cultured on control PU significantly decreased from Day 1 to Day $3(p<0.05)$; no difference was observed between Day 3 and Day 7. For PU coated with gelatin hydrogel a trend similar to the one observed for control PU was detected; in case of PU_GEL15 no significant differences were observed comparing MS1 viability at the three time-points.

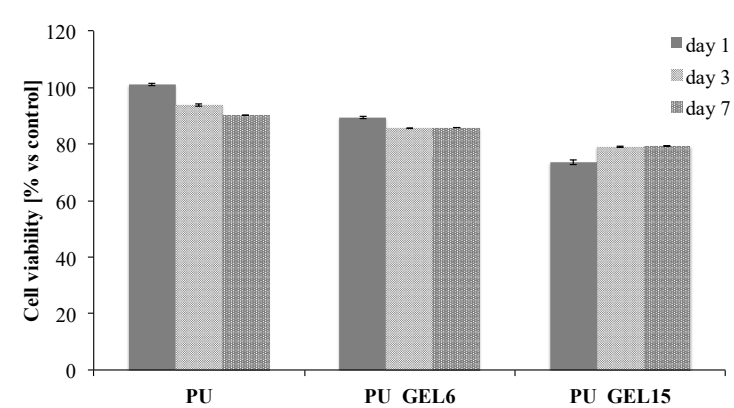

(a)

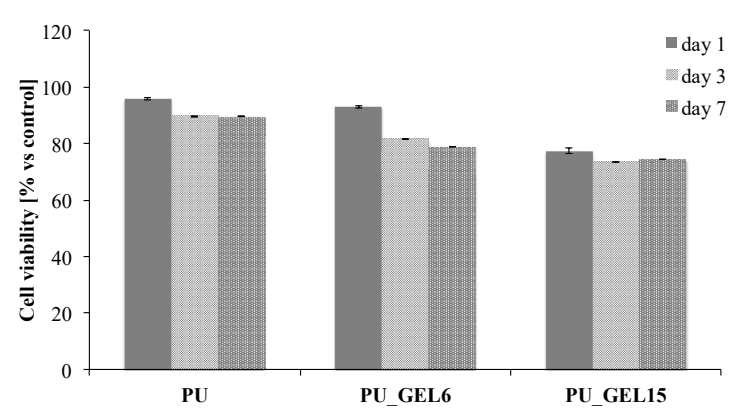

(b) 

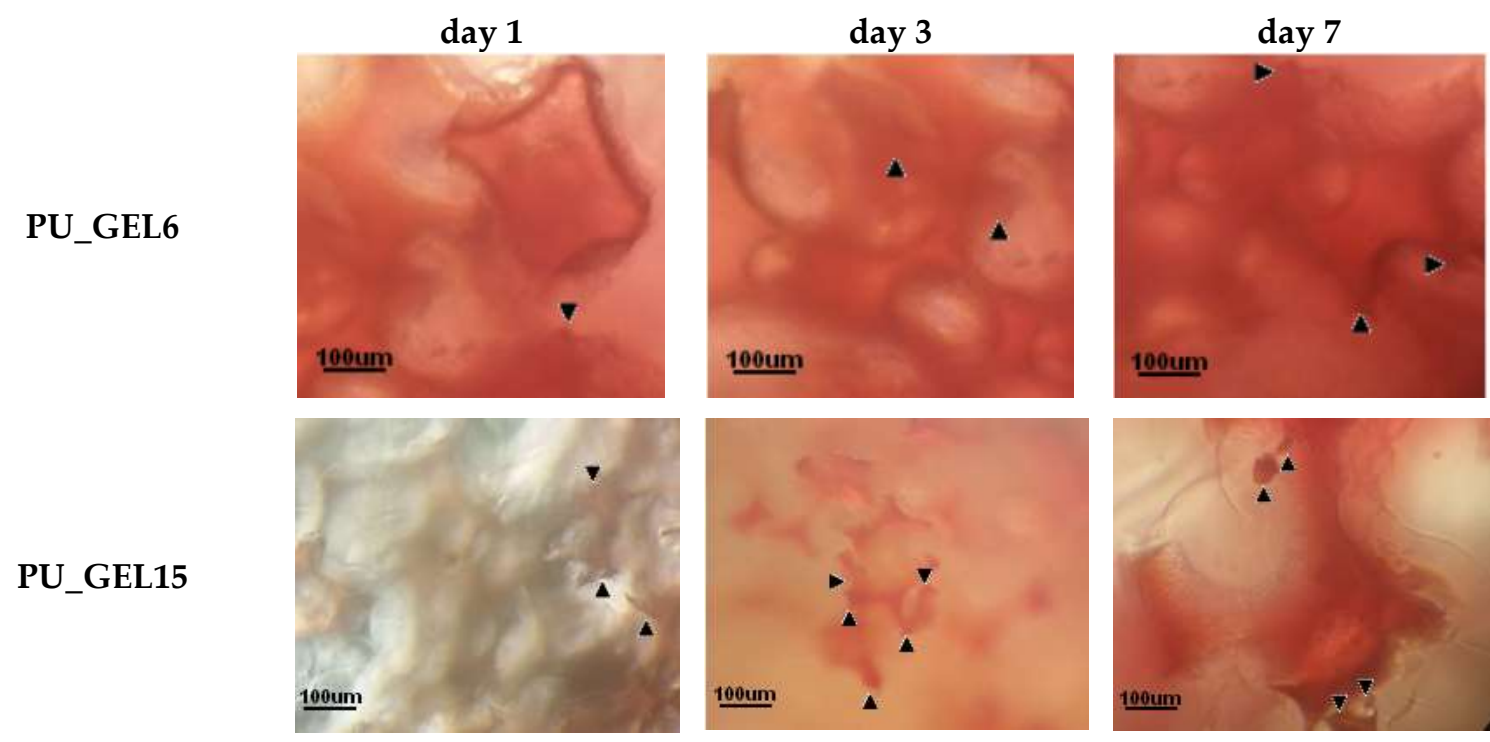

(c)

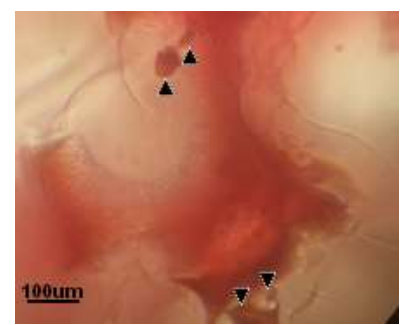

Figure 6. XTT assay performed at the considered time-points for (a) ADSCs and (b) MS1 cells cultured onto control PU, PU_GEL6 and PU_GEL15. (c) Oil Red O staining on PU_GEL6 and PU_GEL15; arrows evidenced lipid vesicles.

\section{PU_CaP}

PU coated with CaPs and control PU, as control, were seeded with AMCs and CMCs to study the possible differentiation into osteoblasts and the influence of the inorganic coating onto the pore walls. In general, it was possible to observe a good cells colonization both onto control PU and coated PU foam for both the tested cell types (Figure 7). Cells were well adherent both on the scaffolds surface and within the PU and PU_CaP pores, thus indicating the absence of cells suffering. SEM results were partly confirmed by histological analysis performed by hematoxylin/eosin staining (Figure 7). At 3 weeks post-seeding, a clear adhesion of AMSCs and CMSCs to the pore surface of the PU scaffold was observed, in particular PU_CaP scaffold. Moreover, cells were observed also in the inner part of the scaffolds, in particular in the case of cells cultured with $\mathrm{NH}$ OsteoDiff medium.

PU

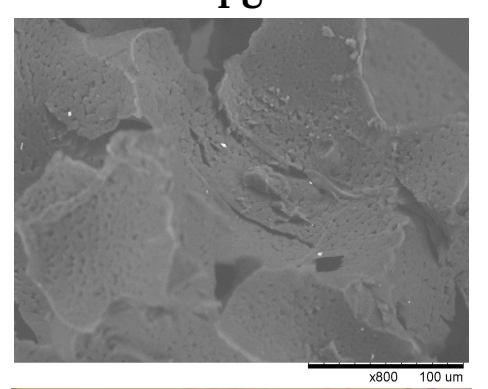

AMCs-EMEM

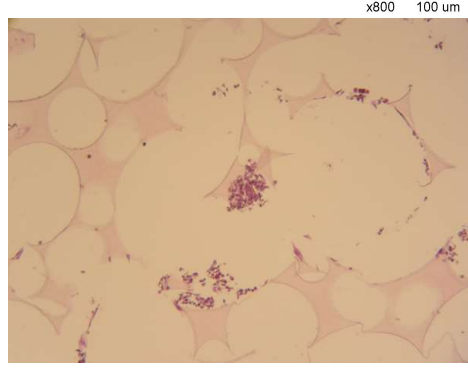

PU_CaP
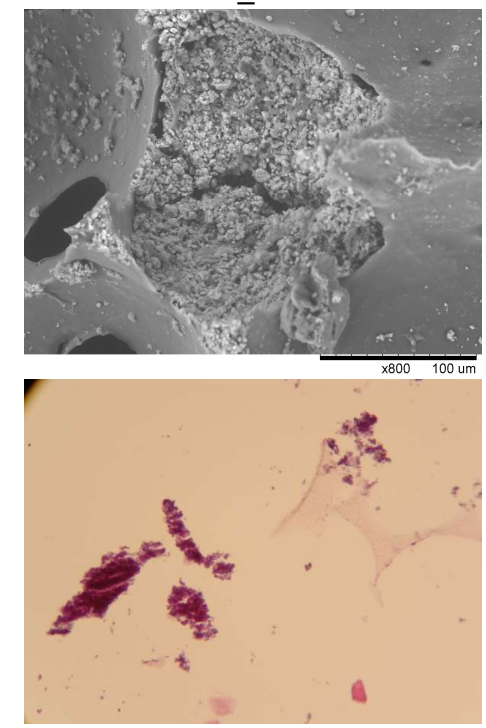


\section{AMCs - NH OsteoDiff}

CMCs-EMEM

\section{CMCs-NH OsteoDiff}
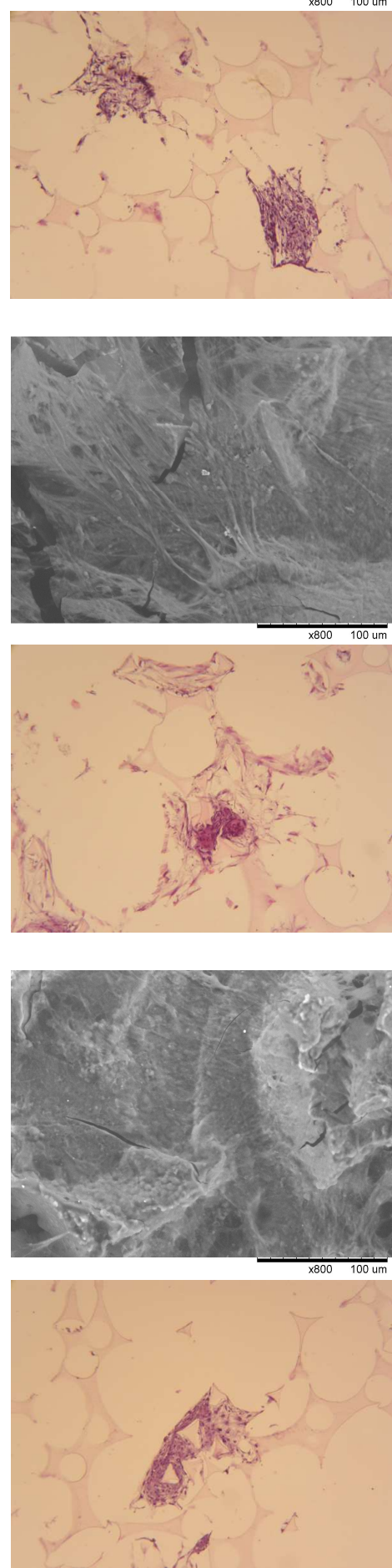
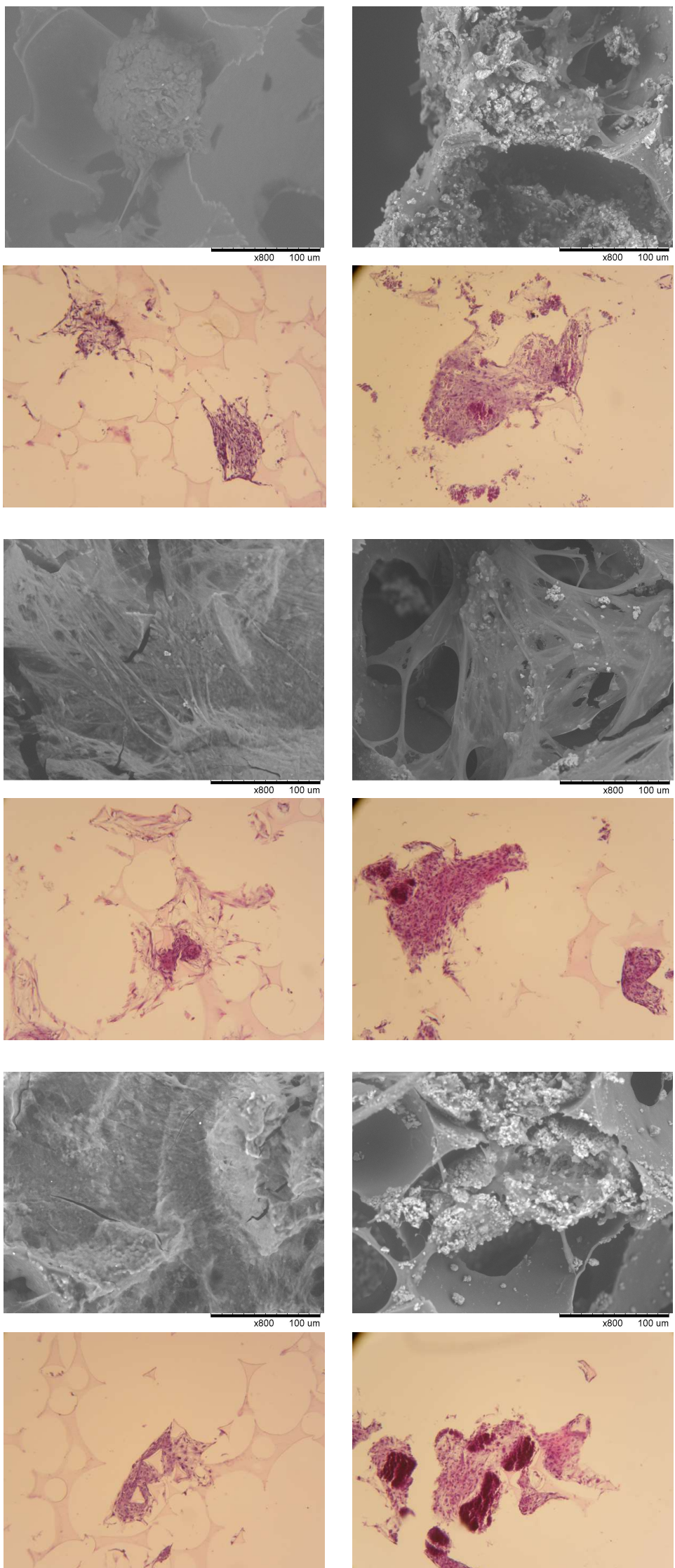

Figure 7. SEM images and hematoxylin/eosin staining of PU and PU_CaP seeded with AMCs and CMCs cultivated in two different culture media. Scale bar: $100 \mu \mathrm{m}$. 


\section{Conclusions}

Polyurethane foam scaffolds were successfully coated by inorganic and organic coatings to respectively target bone tissue and soft tissue regeneration. The morphological analyses showed an effective coating by CaPs, to mimic the inorganic bone component, and gelatin hydrogel, to improve cells adhesion. The produced biofunctionally coated PU scaffolds showed adequate mechanical properties for hard and soft tissue regeneration, depending on the PU coating type and PU foam properties. Cells successfully adhered and proliferated on the scaffolds, showing the correct functionality in depositing inorganic extracellular matrix or in accumulating lipid droplets for bone and soft tissue regeneration, respectively.

Conflicts of Interest: The authors declare no conflict of interest.

\section{References}

1. Tallawi, M.; Rosellini, E.; Barbani, N.; Cascone, M.G.; Rai, R.; Saint-Pierre, G.; Boccaccini, A.R. Strategies for the chemical and biological functionalization of scaffolds for cardiac tissue engineering: A. review. J. $R$ Soc. Interface 2015, 12, 20150254.

2. Chahal, S., Jahir Hussain FS, Kumar, A., et al. Electrospun hydroxyethyl cellulose nanofibers functionalized with calcium phosphate coating for bone tissue engineering. RSC Adv. 2015, 5, 2949729504.

3. Poh, P.S.P.; Hutmache, D.W.; Holzapfel, B.M., et al. In vitro and in vivo bone formation potential of surface calcium phosphate-coated polycaprolactone and polycaprolactone/bioactive glass composite scaffolds. Acta Biomater. 2016, 30, 319-333.

4. He, C.; Xiao, G.; Jin, X., et al. Electrodeposition on nanofibrous polymer scaffolds: Rapid mineralization, tunable calcium phosphate composition and topography. Adv. Funct. Mater. 2010, 20, 3568-3576.

5. Wang, J.; Li, D.; Li, T., et al. Gelatin tight-coated poly(lactide-co-glycolide) scaffold incorporating rhBMP2 for bone tissue engineering. Materials 2015, 8, 1009-1026.

6. Safaeijavan, R.; Soleimani, M.; Divsalar, A., et al. Biological behavior study of gelatin coated PCL nanofiberous electrospun scaffolds using fibroblasts. J. Paramed. Sci. 2014, 5, 2008-4978.

7. Jóźwiak, A.B.; Kielty, C.M; Black, R.A. Surface functionalization of polyurethane for the immobilization of bioactive moieties on tissue scaffolds. J. Mater. Chem. 2008, 18, 2240-2248.

8. De Mulder, E.L.W.; Hannink, G.; Koens, M.J.W., et al. Characterization of polyurethane scaffold surface functionalization with diamines and heparin. J. Biomed. Mater. Res. Part. A 2013, 101A, 919-922.

9. Angeloni, V.; Contessi, N.; de Marco, C., et al. Polyurethane foam scaffold as in vitro model for breast cancer bone metastasis. Acta Biomater. 2017, 63, 306-316.

10. Bertoldi, S.; Farè, S.; Denegri, M., et al. Ability of polyurethane foams to support placenta-derived cell adhesion and osteogenic differentiation: Preliminary results. J. Mater. Sci. Mater. Med. 2010, 21, 1005-1011.

11. Contessi Negrini, N.; Tarsini, P.; Tanzi, M.C., et al. Chemically crosslinked gelatin hydrogels as scaffolding materials for adipose tissue engineering. J. Appl. Polym. Sci. 2019, 136, 47104.

12. Tanzi, M.C.; Farè, S.; Gerges, I. Crosslinked Gelatin Hydrogels. Eur. Patten WO2012164032A1, 6 December 2012.

(C) 2019 by the authors. Licensee MDPI, Basel, Switzerland. This article is an open access article distributed under the terms and conditions of the Creative Commons Attribution (CC BY) license (http://creativecommons.org/licenses/by/4.0/). 